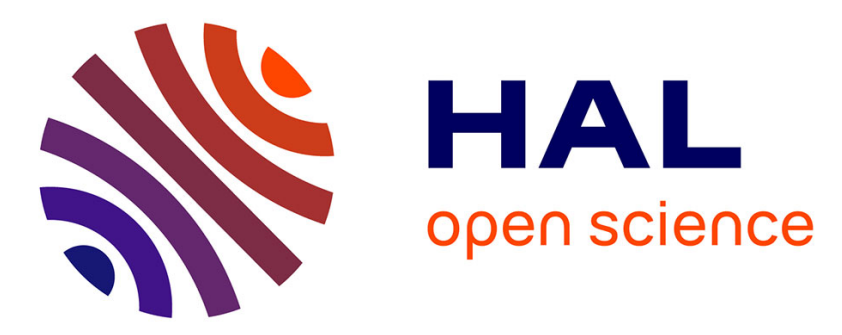

\title{
Test of sampling methods to optimize the calibration of vine water status spatial models
}

A. Herrero Langreo, B. Tisseyre, J.M. Roger, T. Scholasch

\section{To cite this version:}

A. Herrero Langreo, B. Tisseyre, J.M. Roger, T. Scholasch. Test of sampling methods to optimize the calibration of vine water status spatial models. Precision Agriculture, 2017, 19 (2), pp.365-37. 10.1007/s11119-017-9523-8 . hal-01731308

\section{HAL Id: hal-01731308 \\ https://hal.science/hal-01731308}

Submitted on 14 Mar 2018

HAL is a multi-disciplinary open access archive for the deposit and dissemination of scientific research documents, whether they are published or not. The documents may come from teaching and research institutions in France or abroad, or from public or private research centers.
L'archive ouverte pluridisciplinaire HAL, est destinée au dépôt et à la diffusion de documents scientifiques de niveau recherche, publiés ou non, émanant des établissements d'enseignement et de recherche français ou étrangers, des laboratoires publics ou privés. 


\title{
Test of sampling methods to optimize the calibration of vine water status spatial models
}

\author{
Ana Herrero-Langreo ${ }^{1,2}$, Bruno Tisseyre ${ }^{2}$, Jean Michel Roger ${ }^{2}$, Thibaut Scholasch $^{1}$ \\ 1. Fruition Sciences SAS., MIBI, 672 rue du Mas de Verchant. Montpellier 34000, France.
}

2. UMR-ITAP/ Montpellier SupAgro - Irstea. 361 rue Jean-François Breton BP 5095, 34196 Montpellier Cedex 5, France

\begin{abstract}
Plant water status is one of the main factors affecting yield and quality in viticulture. Nevertheless, it is generally difficult to characterize it with enough precision for management purposes. In addition to its temporal variation, related to climate conditions, it has been shown that it is also spatially variable within the vineyard. In practical terms, this makes traditional reference measurements both too costly and time consuming to be affordable. In contrast, it has been shown that spatial variation of plant water status can be inferred from more accessible information, such as plant vigour in Mediterranean conditions. The main practical limitation for this approach is that the relationship between vigour measurements and plant water status is specific for each block and needs to be explicitly calibrated. Furthermore, a high number of measurements are usually required for this calibration.
\end{abstract}

The objective of this work was to propose and test sampling methods to optimize the calibration of a specific spatial model of vine water status using the minimum number of measurements. Two modelbased sampling methods commonly used in non-spatial modelling, Kennard and Stone (K\&S) and Surface Response (SR) were considered, tested and discussed. Satisfactory results were obtained with both methods: with a sample size of 9 calibration sites, both sampling methods gave similar errors to the reference model (Root Mean Standard Error of Prediction, RMSEP=0.1MPa), which was calibrated with 49 sites. Taking into consideration the advantages and limitations of each method, K\&S is considered to be better adapted for the case study presented.

The proposed sampling approach could be extended to other spatial models used in precision agriculture in which ancillary variables can be used to explain most of the spatial variation for any agronomic information of interest.

Keywords Viticulture, sampling, plant water status, water deficit, spatial modelling. 


\section{Introduction}

Plant water status has a large impact on yield and wine quality. In semi-arid conditions, water availability is the main factor regulating berry growth and sugar accumulation (Santesteban and Royo 2006); it is related to other grape quality parameters, such as acidity, phenols composition (Santesteban et al. 2011) and flavonoids content (Zarrouk et al. 2012).

The most common reference measurements of vine water status are leaf water potential (LWP), stem water potential (SWP) and predawn leaf water potential (PLWP), all of which are manually taken using pressure chambers (Girona et al. 2006; Scholander et al. 1965; Sibille et al. 2007). As such, the procedure is slow, costly and requires skilled labor. As an additional difficulty, plant water status presents important spatial variation within the block and evolves rapidly through the season (Acevedo-Opazo et al. 2008; Taylor et al. 2010). As a result, plant water status is difficult to characterize for management purposes, not only due to economic and time constraints, but also because of practical limitations.

As an alternative, Kazmierski et al. (2011) and Tisseyre et al. (2008) showed that spatial patterns of vigour in non-irrigated Mediterranean conditions are temporally stable. In fact, Acevedo-Opazo et al. (2010) used this assumption to estimate spatial and temporal variations of plant water status under similar conditions. In this work, spatial variations of plant vigour indices (trunk circumference and exposed leaf area), measured on a single date were used to estimate the relative spatial variations of plant water status, as compared to a reference site. Therefore, in order to estimate the spatial and temporal variations of plant water status on a 1.2 ha block during 13 dates, throughout two seasons, plant vigour measurements obtained in a single date across the block were combined with plant water status measured at a single reference site over time.

There is, nevertheless, an important practical limitation to this approach: the relationship between vigour measurements and plant water status is block specific; it depends upon field conditions and vineyard characteristics, and it needs to be explicitly calibrated. As a consequence, a large number of plant water status measurements are required in order to calibrate the model of a particular block. For instance, predawn leaf water potential had to be measured at 49 sites over 9 dates by Acevedo-Opazo et al. (2010), resulting in 441 measured sites used to study plant water status on a 1.2 ha block.

This is a common problem in precision agriculture. For different types of agronomic information, the main reported limitation for mapping information is that the cost of intensive sampling would be greater than the benefits gained (Bongiovanni and Lafayette 2004; Bramley et al. 2011; Heiniger et al. 2003). Consequently, linear regression (LR) models are usually used to estimate time consuming and/or costly information from related ancillary data (AD), which is cheaper and/or easier to measure. In the literature, some examples can be found, like the use of the normalised difference vegetation index (NDVI) to estimate plant vigour, canopy density and size; as well as production quality (Dutta 2013; Johnson 2003; Samani Majd et al. 2013) and/or near infra-red spectral images used to evaluate nitrogen status (Kyveryga et al. 2011; Onoyama et al. 2015). Such an approach has also been used in soil studies relating soil electrical conductivity to soil concentrations of selected mineral elements (Heiniger et al. 2003) or water content (Corwin and Lesch 2005).

The advantage of using a regression model is that, theoretically, it can be calibrated with a very small number of measurements. A set of $q+1$ sites theoretically allows an ordinary least squares regression to calibrate a linear model between $\mathrm{q}$ input variables and the response (Craven and Islam, 2011). Consequently, as suggested by Acevedo et al, (2010), an appropriate site selection (i.e, sampling) could allow the calibration of a LR model based on 2 input variables with as few as 3 to 10 sites per block, as long as the sites are representative of the relationship between the considered variables.

Wulfsohn (2010) presented a review on sampling for precision agriculture that summarized the most common methods for gathering the spatial variability of a certain variable at the block level. However, few studies have focused on how to optimize site selection for LR spatial models based on model input 
variables. Lesch et al. (1995) suggested a method based on surface design modification. This method, called pseudo-surface response, yielded a significant reduction of the number of sampling sites (from 198 to 16 sites per model calibration, applied over $13.7 \mathrm{ha}$ ) without altering significantly the quality of the model calibration. Hengl et al. (2003) compared a stratified sampling approach with a surface response sampling approach to calibrate a spatial model. The approaches described by Lesch et al. (1995) or Hengl et al. (2003) were applied to study soil variability.

The aim of this work was to propose and test two sampling procedures commonly used to optimize the calibration of ordinary LR models, Kennard and Stone (Kennard and Stone 1969) and a Surface Response method (Lesch et al. 1995; Box and Draper 1986), for calibrating the spatial vine water status model proposed by Acevedo et al. (2010) with the minimum number of measurements.

The following three constraints were considered as criteria for the selection of the sampling methods:

i) Minimize sample size, so that the model calibration could be done with as few as 3 to 10 sites per block.

ii) Select sampling sites using the information provided by the input variables of the model, i.e. ancillary data $(\mathrm{AD})$ regarding vigour measurements.

iii) Account for several input variables, in order to integrate the available information of $\mathrm{AD}$ variables, in this case trunk circumference and exposed leaf area, for site selection.

These sampling specifications could be extended to other spatial models used in precision agriculture. To the best of the authors' knowledge, such an approach has never been reported in the literature.

\section{Materials and methods}

\section{Sampling procedures}

In order to minimize the sample size needed for calibration, two model-based sampling methods were considered, i.e. methods focused on identifying the sites best representing the model relationship between the ancillary data $(\mathrm{AD})$ and the agronomic information, plant water status. The sampling methods considered in this study were Kennard and Stone (K\&S), first described by Kennard and Stone (1969), and a surface response (SR) approach, as described by Lesch et al. (1995). Both methods require exclusively $\mathrm{AD}$ to select the calibration sites and can account for several auxiliary variables simultaneously.

The sites or locations selected by each method for model calibration are called hereafter 'calibration sites'. The set of 'calibration sites' is called a 'sample'; the number of sites in the sample is the 'sample size'.

$\mathrm{K} \& \mathrm{~S}$ is a common sampling method used in chemometric applications. It is based on experimental design theory and aimed at LR models. The approach of the method is to select calibration sites that maximize the variance gathered by the sample in the feature space, that is, according to their values on the AD.

In turn, SR sampling approaches are specifically designed for controlled experimental conditions (Box and Draper 1986). These sampling methods propose different sampling schemes according to the type of model and distribution of the experimental data. This is an advantage compared to K\&S, as SR methods can be adapted to several kinds of models, including non-linear models. SR methods also take into account the data distribution to exclude outliers. In the following, a response sampling scheme, applied by Lesch et al. (1995) and Hengl et al. (2003) is considered.

\section{$\underline{\text { Kennard and Stone }(K \& S)}$}


First described by Kennard and Stone (1969), the K\&S method starts with a list of candidate sites, all available sites that could be potentially used for model calibration, and their AD co-ordinates, which in this case are the values of the corresponding AD variables. AD values were normalized by subtracting the mean $\mathrm{AD}$ of all candidate sites and dividing by the standard deviation. The site with the closest value to the centroid of $\mathrm{AD}$ in the dataset is used as an initialization site (Site 0). The furthest point from Site 0 in terms of $\mathrm{AD}$ values is then selected and included into the calibration sample. In further iterations, the distance between the sample subset (the group of sites already selected) and each of the remaining candidate sites is computed as the minimum distance between any of the sites in the sample and the candidate site.

After each subsequent iteration, the algorithm selects the candidate site that exhibits the largest distance to the sample already selected. Such a procedure is repeated until the sample size specified by the analyst is achieved.

\section{Surface Response (SR)}

Surface response (SR) methodology is a mathematical technique for empirical model building that aims at relating explanatory variables to a response variable of interest. Originally developed by Box and Draper to build optimal experimental designs (Box and Draper 1986), SR is now used in numerical modeling. As a first step, SR methods determine the best set of data to build the model. A SR design specifies the values of $\mathrm{AD}$ that the calibration sites should have in order to obtain the best possible calibration of the model ("design levels"). Depending on the phenomenon to be modeled, numerous schemes could be chosen. For linear models applied to spatial data, Lesch et al. (1995) and Hengl et al. (2003) selected the second-order, rotatable central composite response surface design (CCRSD) (Lesch et al. 1995).

CCRSD was selected as SR design in the current work. This method is designed to minimize the number of calibration sites for multivariate LR models (Lesch et al., 1995). Hereafter, SR method refers to the surface response method proposed by these last authors.

For CCRSD, the ancillary database is transformed into a normalized and de-correlated matrix (scores) via a principal components analysis (PCA). The CCRSD specifies eight design levels located at a distance of 1.96 from the centre of the population in a normalized PCA plane and one at the centre of the plane (Figure 1). When the sample size was restricted to 4 sites, the four "design levels" on the main axis of the PCA axis were used for model calibration. When the sample size was restricted to 5 sites, the closest site to the average value of the population was also included. The same scheme was applied to sample sizes of 8 and 9 sites, including "design levels" on the main axis of the PCA as well as on their bisectrices.

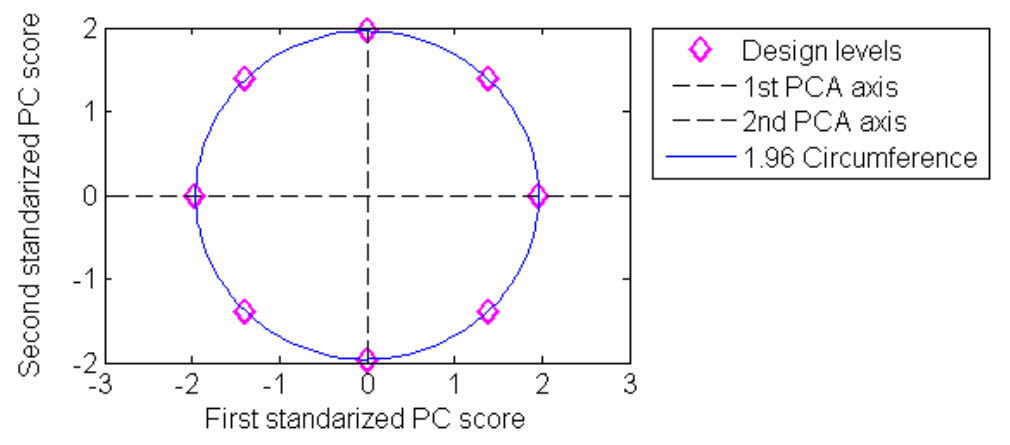

Fig. 1. Design levels corresponding to a second-order rotatable central composite response surface design (CCRSD).

Finally, sites with ancillary values closest to the pre-defined "design levels" specified by the SR design were selected for model calibration.

This sampling scheme implies that sample size can only be $4,5,8$ or 9 sites. 


\section{Evaluation of the sampling methods}

The performance of each sampling method at selecting the best sampling sites for model calibration was assessed based on the root mean standard error of prediction (RMSEP). It represents the error when applying the calibrated model to estimate PLWP in sites where only AD measurements are available.

The RMSEP was computed as follows:

$$
R M S E P=\sqrt{\frac{\sum_{i=1}^{n}\left(z_{\text {pred }}\left(s_{i}\right)-z_{\text {obs }}\left(s_{i}\right)\right)^{2}}{n}}
$$

Where $n$ is the number of calibration sites selected by each sampling method (sample size).

$Z_{o b s}\left(S_{i}\right)$ are the observed values of the PLWP for each site $\left(S_{i}\right)$

$Z_{\text {pred }}\left(S_{i}\right)$ are the values of PLWP predicted by the model for each site $\left(S_{i}\right)$

The RMSEP obtained with each sampling method was compared with two error values obtained from the reference model:

$$
\begin{aligned}
& \text { - the standard deviation of the observed PLWP (SD) and } \\
& \text { - the root mean standard error of calibration (RMSEC). }
\end{aligned}
$$

The reference model is the calibrated model using PLWP measured exhaustively all over the block with the same resolution as the $\mathrm{AD}$.

The SD was used to characterize the error when the average PLWP value (obtained after the block is measured exhaustively) is used as the estimated PLWP value for every site. Thus, SD indicates the average error produced by neglecting/discarding the spatial variation within the block.

The RMSEC is the error obtained when PLWP measured exhaustively within the block is used for calibrating the model. It represents the average error expected for the spatial model, simply due to estimating PLWP through a model based on AD.

The RMSEC was computed as follows:

$$
R M S E C=\sqrt{\frac{\sum_{i=1}^{n}\left(z_{e s t}\left(s_{i}\right)-z_{o b s}\left(s_{i}\right)\right)^{2}}{n-p-1}}
$$

Where $n$ is the number of sites in the block (49 sites).

and $p$ is the number of variables of the LR model

$Z_{\text {obs }}$ are the observed values of the PLWP for each site $\left(S_{i}\right)$

$Z_{e s t}$ are the values of PLWP estimated from the reference model for each site $\left(S_{i}\right)$

\section{Modelling}

According to the work by Acevedo-Opazo et al., (2010), a linear regression model based on two AD, trunk circumference $(T C)$ and exposed leaf area (ELA), was applied to estimate plant water status (PLWP) (Eq. 3). 


$$
z\left(s_{i}\right)=b 0+b 1 * T C\left(s_{i}\right)+b 2 * E L A\left(s_{i}\right)
$$

Where Si refer to georeferenced locations in the block for which AD and PLWP were measured $\hat{z}\left(s_{i}\right)$ refers to PLWP values estimated for each $S_{i}$

$b 0, b 1$ and $b 2$ are coefficients of the model to be determined by the calibration.

\section{Field measurements}

Vine water status was measured as predawn leaf water potential (PLWP) between 3:00 and 5:00 a.m. using a pressure chamber (Scholander et al. 1965) on a date corresponding to significant water restriction. Three adjacent vines were measured at each sampling site and averaged to obtain a site value $\mathrm{Z}\left(S_{i}\right)$.

$\mathrm{AD}$ were based on manual field observations avoiding non-representative plants (showing disease symptoms, for example) and information averaged over 5 plants. This measurement method implies that this AD is very reliable. In addition, these variables are usually easier to obtain than the PLWP.

Exposed leaf area (ELA) was measured manually using the method of Murisier and Zufferey (1997) Trunk circumference $(T C)$ was measured $100 \mathrm{~mm}$ above the graft of each target vine.

The collection methods and data sources are summarized in Table 1.

Table 1. Summary of the variables measured, nomenclature, units and acquisition dates.

\begin{tabular}{|c|c|c|c|}
\hline Variable & Nomenclature & Units & Acquisition dates \\
\hline $\begin{array}{lll}\text { Pre-dawn Leaf water } \\
\text { potential }\end{array}$ & PLWP & $\mathrm{MPa}$ & August 2003 \\
\hline Exposed Leaf Area & ELA & $\mathrm{m}^{2}$ & August 2003 \\
\hline Trunk circumference & $\mathrm{TC}$ & $\mathrm{cm}$ & March 2006 \\
\hline
\end{tabular}

Acevedo et al (2010), Tisseyre et al. (2008) and Kazmierski et al. (2011) highlighted the temporal stability, over 10 years, of within-block vigour patterns and their relationship with plant water status in Mediterranean conditions. In this context, the use of data from different seasons in the model is considered to be appropriate for this particular case. TC is considered as an integrative attribute taking into account the average vigour of the vines from the date of plantation, while ELA accounts for the seasonal vegetative growth, spatially determined by such vigour patterns.

\section{Block description}

The study domain was a 1.2 ha Syrah block located at the experimental vineyard of INRA Pech-Rouge (43.1414 N, 3.1314 E, WGS84; Gruissan, Aude, France). The block was non-irrigated, established in 1990 with $1 \mathrm{~m}$ spacing between vines and $2.5 \mathrm{~m}$ spacing inter-rows and trained in a vertical shoot positioning system. The block exhibited significant soil variability. Its geological formation was composed of interbedded micritic limestone with important accumulations of red clay in some parts of the block (Coulouma et al. 2010) which induce large differences in soil water availability and consequently vine growth. All variables were available on 49 sites (Figure 2), displayed in a systematic grid. Each site is associated with a single geo-referenced position in the block. The value reported for each site resulted from the average of measurements performed in 5 vines. 


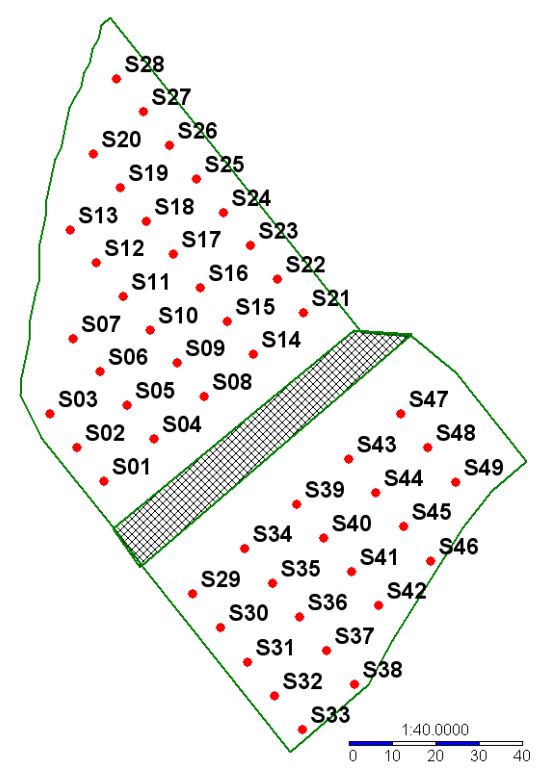

Fig. 2. Locations of measurement sites within the block for all AD and PLWP. Key scale is expressed in meters.

\section{Mapping}

Mapping was done with the 3D-field software (Version 2.9.0.0, Copyright 1998-2007, Vladimir Galouchko, Russia). In order to facilitate the mapping, inverse distance weighting was used for interpolation. Map legends can be interpreted according to the PLWP classes defined by Ojeda et al. (2005): (i) no water restriction (0>PLWP> $-0.2 \mathrm{MPa}$ ), (ii) low water restriction (-0.2>PLWP>-0.4 MPa), (iii) medium water restriction $(-0.4>\mathrm{PLWP}>-0.6 \mathrm{MPa})$, (iv) high water restriction $(-0.6>\mathrm{PLWP}>-1.0$ $\mathrm{MPa})$ and (v) severe water restriction (PLWP<-1.0 MPa).

\section{Results}

Differences in TC and ELA values of the calibration sites selected by each method are illustrated in Figure 3. This plot shows normalized TC and ELA for all 49 sites of the block. As an example, sites selected by both methods for a sample size of 5 are labelled in the figure. Site numbers correspond to the site locations indicated in Figure 2. Both methods selected calibration sites near the edges of the range of TC and ELA values. SR avoided far-out values and included a site with the closest value to the mean (S17).

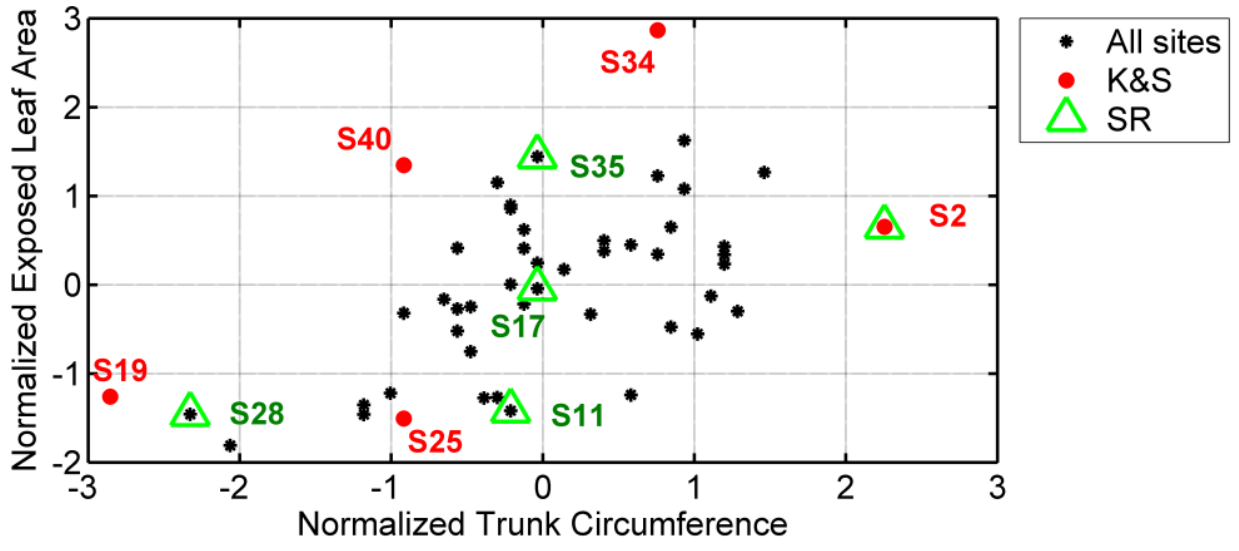

Fig. 3. Calibration sites selected by each method, for sample sizes of 5 sites. 
Concerning the spatial distribution, neither of the two sampling methods selected sites evenly distributed over the block. Instead, for a sample size of 5 calibration sites, both K\&S and SR selected sites located at the driest and at the most humid regions of the block: a severe water restriction area at the northern part and a no water restriction area at the south western part of the block (Fig. 4). An additional site, at the western part of the block was also selected by both methods. This site, S2, presented a high but not too severe water restriction and the highest TC value for an average ELA.

a)

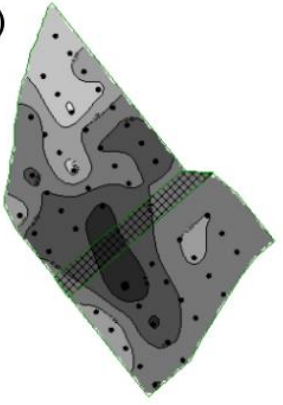

b)

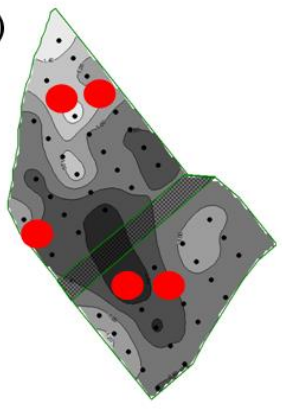

c)

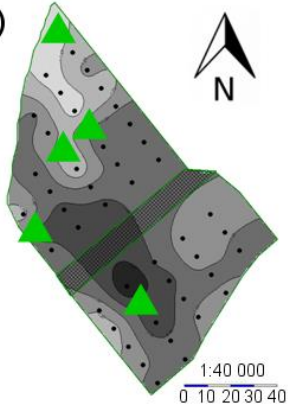

PLWP (MPa)

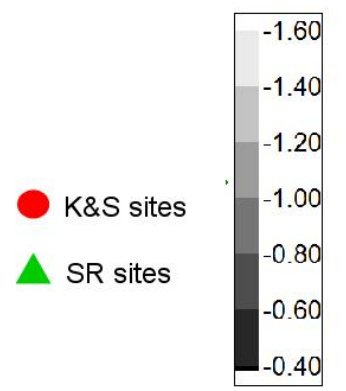

Fig. 4. Observed (a) and estimated PLWP values using the spatial model calibrated with 5 sites selected through K\&S (b), and 5 sites selected through SR (c). Key scale is expressed in meters.

Regarding PLWP zones, K\&S selected sites grouped in pairs at locations corresponding to extreme water restrictions zones while SR included less sites in those extreme zones, but added 2 sites in a medium water restriction region. This result is consistent with the PLWP values of the selected samples observed in Figure 6.

Figure 5 plots the results of model calibration for each method. The RMSEP of the models built with 3 to 15 calibration sites, selected by either K\&S or SR. Note that K\&S can be used for any sample size whilst SR only allows selection of $4,5,8$ or 9 calibration sites.

For both methods, RMSEP was lower than $0.15 \mathrm{MPa}$ for any sample size. Furthermore, when using over 9 calibration sites, both methods provided models with a similar error to the reference model, RMSEC $_{\text {ref }}$ (i.e. the calibration error of the spatial model using all the 49 available measurements of PLWP over the block).

As expected, the RMSEP decreased for larger sample sizes, especially in the case of SR models, which dropped from $0.14 \mathrm{MPa}$ with 5 sites, to the $\mathrm{RMSEC}_{\text {ref }}$ value $(0.10 \mathrm{MPa})$ when 8 sites were used for calibration. RMSEP for K\&S method showed better results for the smallest sample sizes (RMSEP $=0.12$ MPa for 5 calibration sites), although, in this case, 9 calibration sites were needed to obtain an error similar to the reference model. 


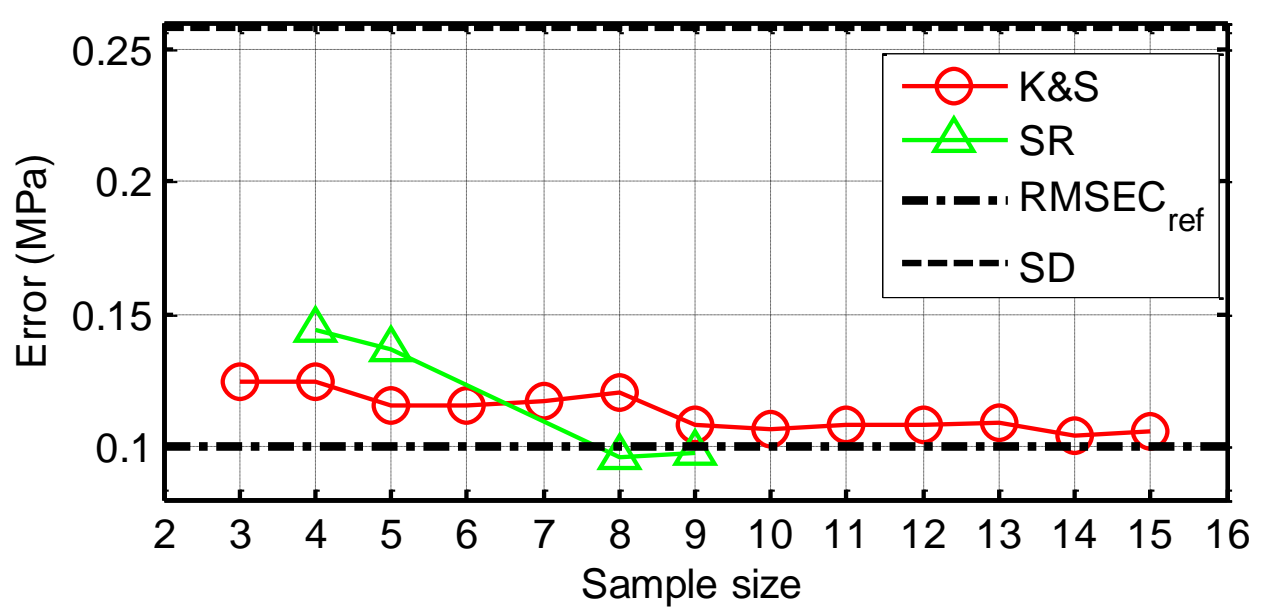

Fig. 5. Root Mean Standard Error of Prediction (RMSEP) of the spatial model calibrated with 3 to 15 sites chosen according to $\mathrm{K} \& \mathrm{~S}$ and SR sampling. Horizontal lines represent respectively the standard deviation (SD) of the population on the target variable $(0.26 \mathrm{MPa})$; and the root mean standard error of calibration $\left(\mathrm{RMSEC}_{\mathrm{ref}}\right)$ on the reference model $(0.10 \mathrm{MPa})$ obtained with all the 49 available sites.

Figure 6, illustrates plant water status measurements (PLWP) of the calibration sample selected by each method for 5 and 8 sites.

In accordance with the AD values, shown in Figure 3, when looking at the PLWP values of the selected samples on the reference model (Figure 5), the sites selected by K\&S are located at the extremes of the range of the observed and estimated PLWP values, while SR sites are closer to the mean.

a)

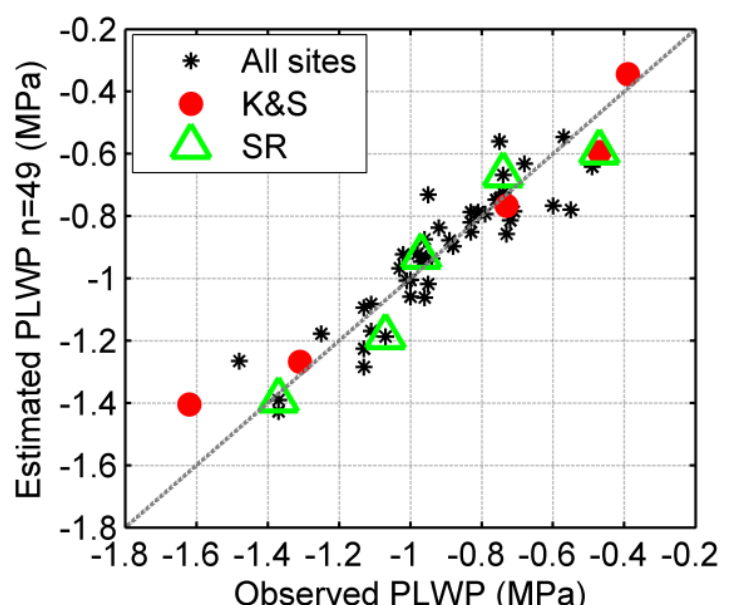

b)

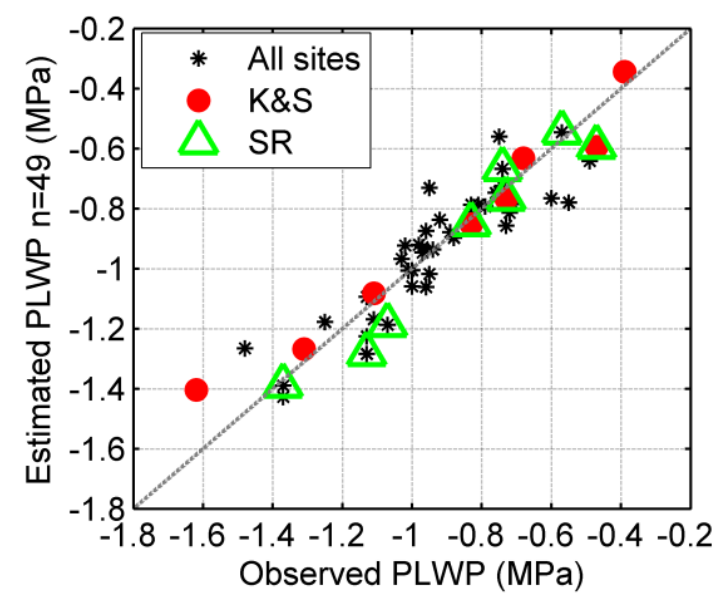

Fig. 6. Sites selected by each method and represented according to observed and estimated values on the reference model, for sample sizes of: a) 5 sites and b) 8 sites

For 5 calibration sites, a better performance of the K\&S method was observed for two reasons: i) the greatest variation range covered, and ii) the lowest dispersion of the points around the bisecting line. In contrast, as shown in Figure 5, when increasing the sample size from 5 to 8 calibration sites, SR method obtained better results. In this case, RMSEP improved for SR method while maintaining similar values for K\&S. When increasing the sample size from 5 to 8 calibration sites, SR represented better the edges of the variation range, avoiding, at the same time, an extreme point with low observed PLWP, overestimated by the reference model. In turn, the K\&S method added 3 sites closer to the mean. In this case, the model calibration did not change substantially from 5 to 8 calibration sites and similar values of RMSEP were obtained. 


\section{Discussion}

Both methods showed good results in terms of RMSEP: with a sample size of 9 calibration sites, both sampling methods had similar errors to the reference model (RMSEP=0.1MPa), which was calibrated with 49 sites.

SR selected sites with closer values to the mean, gathering less of the data variability and avoiding extreme values. In contrast, $K \& S$ maximized the variance covered by the calibration sites and yielded better results with the smaller datasets ( 5 or less calibration sites). The performance difference between both methods can be related to the nature of the AD used in the case study. The dataset of AD comprised 49 TC and ELA values, obtained from averaged measurements and avoiding non-representative plants, in order to assure the reliability of the data. An atypical point, over-estimated by the reference model (shown in Figure 6), was observed at the lowest edge of the range. These features determined the main difference between sampling methods. Assuming the representativity of the data at the extremes of the model, for LR models, optimum calibration was provided by a sample set including the largest variation both in the input and output variables of the model (Hengl et al. 2003). In this regard, K\&S aims at gathering the maximum variability on the sampled dataset and assumes the reliability of all candidate sites considered eligible for calibration. Conversely, SR takes into account the distribution of the AD to optimize the site selection. SR assumes a normal distribution of the data, selects representative sites according to such distribution and specifically excludes limiting sites with $\mathrm{AD}$ outside of a certain confidence interval (outside $95 \%$ of the population). Hence, SR reduces the variability gathered by the sample but allows excluding atypical values easily. As a consequence, the $\mathrm{K} \& \mathrm{~S}$ method provided a better performance for smaller sample sizes, as it was able to cover a greater variation range with fewer samples. Meanwhile, for bigger sample sizes, SR was able to cover the variation range of the model, while excluding an atypical site with low observed PLWP values (Figure 6). SR provided in this case slightly better results than the $\mathrm{K} \& S$ method (for 8 calibration sites, $\mathrm{RMSEP}_{\mathrm{SR}}=0.09 \mathrm{MPa}, \mathrm{RMSEP}_{\mathrm{K} \& \mathrm{~S}}=0.12 \mathrm{MPa}$ ).

SR relies on a normal distribution, which is an assumption that becomes less suitable with smaller datasets. As an illustration, the selection of the SR "design levels" and their closest selected sites are plotted in Figure 7. It can be observed that the AD of some of the selected sites are quite distant from the pre-defined design levels (up to 1.18 normalized units). This observation points to the K\&S method as most advisable for the case study presented. Conversely, SR could be considered for more exhaustive AD datasets.

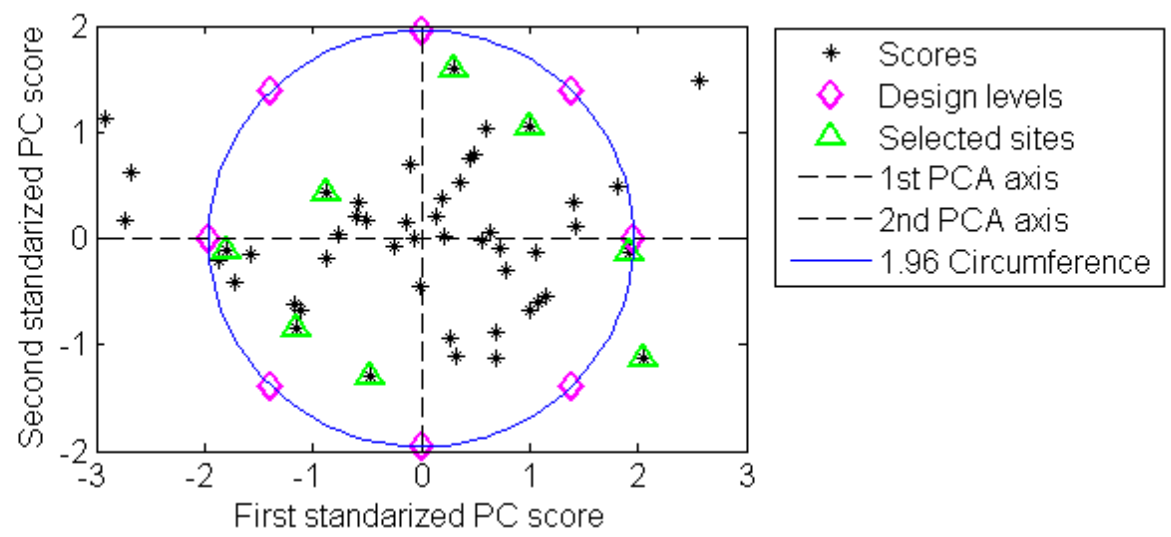

Fig. 7. A second-order CCRSD overlaid onto transformed and decorrelated AD, for the purpose of identifying calibration sites.

As a conclusion, for reference models with similar errors (RMSEC), SR is expected to be better adapted to high resolution ancillary measurements (such as NDVI images), composed of large datasets, rather than to medium or low resolution measurements, for which the available datasets tend to be small. In contrast, $\mathrm{K} \& \mathrm{~S}$ is affected by the reliability of the data, especially at the extremes of the model, rather than by the size of the dataset. This method might be more appropriate for carefully pre-processed, low 
resolution datasets, where outliers have been already discarded and the reliability of AD improved by an averaging or filtering process.

In addition, as the proposed methods focus solely on the $\mathrm{AD}$ values of the sites and not on their location, the calibration sites were spatially close to each other, in particular for the K\&S method, and yet a low RMSEP was obtained for such calibrations. This result implies that most of the spatial variation in PLWP was well explained by ELA and TC without needing to take into account the location of the sites. As formally demonstrated by Lesch (2005), LR sampling methods can be applied to spatial data as long as it can be assumed that most of the spatial variation of the response variable estimated by the model can be explained by the AD.

In future work, considering the spatial location of the data could allow minimizing the impact of erroneous measurements when using the K\&S sampling method. Each calibration site, sequentially selected by the method, could be verified according to its location and its concordance to the spatial variance of surrounding sites. Such an improvement in the approach would exclude outlier sites not representative of their environment.

Other practical aspects to take into account for the choice of a sampling method depend on the expert agronomic knowledge and data already available. The K\&S method can be used to best complement an existing database of already available measurements, taken on site locations selected by any other sampling method. Moreover, as the K\&S method provides a rank indicating in which order the sites need to be sampled to optimize model calibration, it allows for the selection of an initial sample, with a given affordable number of sites, and later on, the addition of new sites to complement the sample.

In turn, the SR method can be used in conjunction with agronomic expert knowledge when selecting the calibration sites. SR sampling selects calibration sites along the axis of a principal components analysis (PCA). In a study relating electrical conductivity and soil properties, Hengl et al.(2003) pointed out that sampling along the axes of a PCA corresponds to sampling along meaningful orthogonal predictors expected to represent soil-forming processes (as soil experts would do intuitively). An advantage of such a procedure is that PCA axes and the location of the "calibration sites" in those axes, can be interpreted according to what the $\mathrm{AD}$ variables mean in terms of agronomic application.

As mentioned in the introduction, the sampling problem addressed in this work is usually found in other applications in precision agriculture. Model calibration is necessary to apply LR models to a specific field, block or management unit. The proposed sampling methods could be used to facilitate a wider application of those models.

\section{Conclusions}

This work is a preliminary study towards the practical application of a spatial prediction model of vine water status. It deals with an important operational constraint: to reduce the number of measurements needed to calibrate the model for a specific block.

The proposed approach is to use model-based sampling techniques, which minimize the number of measurements needed for calibration, by selecting the samples best describing, not the whole available dataset, but the relationship between the variables of the model.

Both sampling methods proposed, Kennard and Stone $(K \& S)$ and the second-order, rotatable central composite response surface design (SR), allowed model calibration with small sample sizes (3 to 15 and 4 to 9 calibration sites, respectively). With a sample size of 9 calibration sites, both methods provided similar performance to the reference model, which was calibrated with 49 sites. K\&S was the preferred sampling method in the case study presented, providing better results with the smaller sample sizes ( $\mathrm{RMSEP}=0.12 \mathrm{MPa}$ with 3 calibration sites and $\mathrm{RMSEP}=0.11 \mathrm{MPa}$ with 5 calibration sites). The main difference between methods is based on the assumptions made regarding the ancillary data used to select 
the calibration sites. While $\mathrm{K} \& \mathrm{~S}$ requires reliable data (absence of outliers), particularly at the edges of the model, SR assumes normal distribution of the data and requires more extensive ancillary data datasets, representing more intensively the whole range of variation used in the model.

Practical aspects to take into account when choosing a sampling method were also observed and discussed. In particular, the potential use of the SR method for the integration of expert agronomic knowledge, as well as the plausible procedures to use the K\&S method for best complementing already available calibration samples, were discussed.

The main limitation so far for the proposed methods is that the locations of the calibration sites are not taken into account.

This is a first, essential step for growers to be able to apply experimental models to spatially optimize management and harvest. 


\section{References}

Acevedo-Opazo, C., Tisseyre, B., Guillaume, S., \& Ojeda, H. (2008). The potential of high spatial resolution information to define within-vineyard zones related to vine water status. Precision Agriculture, 9(5), 285-302.

Acevedo-Opazo, C., Tisseyre, B., Ojeda, H., \& Guillaume, S. (2010). Spatial extrapolation of the vine (Vitis vinifera L.) water status: a first step towards a spatial prediction model. Irrigation Science, 28(2), 143-155.

Bongiovanni, R., \& Lafayette, W. (2004). Precision Agriculture and Sustainability. Precision Agriculture, 5, 359-387.

Box, G. E. P., \& Draper, N. R. (1986). Empirical Model-Building and Response Surface. New York, NY, USA.: John Wiley \& Sons, Inc.

Bramley, R. G. V, Ouzman, J., \& Thornton, C. (2011). Selective harvesting is a feasible and profitable strategy even when grape and wine production is geared towards large fermentation volumes. Australian Journal of Grape and Wine Research, 17(3), 298-305.

Corwin, D. L., \& Lesch, S. M. (2005). Apparent soil electrical conductivity measurements in agriculture. Computers and Electronics in Agriculture, 46(1-3), 11-43.

Coulouma, G., Tisseyre, B., \& Lagacherie, P. (2010). Is a systematic two dimensional EMI soil survey always relevant for vineyard production management? A test on two pedologically contrasting Mediterranean vineyards. In R. A. V. Rossel, A. B. McBratney, \& B. Minasny (Eds.), Proximal soil sensing. Progress in soil science series. Heidelburg, Germany: Springer.

Craven, B. D., \& Islam, S. M. (2011). Ordinary least-squares regression. In Moutinho, L.; Hutcheson, G. D. (Ed. ), The SAGE Dictionary of Quantitative Management Research (pp. 224-228). Bangalore, India: SAGE Publications.

Dutta, R. (2013). Monitoring green leaf tea quality parameters of different TV clones grown in northeast India using satellite data. Food Chemistry, 139(1-4), 689-694.

Girona, J., Mata, M., Del Campo, J., Arbonés, a., Bartra, E., \& Marsal, J. (2006). The use of midday leaf water potential for scheduling deficit irrigation in vineyards. Irrigation Science, 24(2), 115127.

Heiniger, R. W., McBride, R. G., \& Clay, D. E. (2003). Using soil electrical conductivity to improve nutrient management. Agronomy Journal, 95(3), 508-519.

Hengl, T., Rossiter, D. G., \& Stein, A. (2003). Soil sampling strategies for spatial prediction by correlation with auxiliary maps. Australian Journal of Soil Research, 41(8), 1403.

Johnson, L. F. (2003). Temporal Stability of the NDVI-LAI Relationship in a Napa Valley Vineyard. Australian Journal Of Grape And Wine Research, 9(2), 96-101.

Kazmierski, M., Glemas, P., Rousseau, J., \& Tisseyre, B. (2011). Temporal stability of within-field patterns of NDVI in non irrigated mediterranean vineyards. Journal International Des Sciences De La Vigne Et Du Vin, 45(2), 61-73.

Kennard, R., \& Stone, L. (1969). Computer Aided Design of Experiments. Technometric, 11(1), 137148. 
Kyveryga, P. M., Blackmer, T. M., \& Pearson, R. (2011). Normalization of uncalibrated late-season digital aerial imagery for evaluating corn nitrogen status. Precision Agriculture. 13(1), 2-16

Lesch, S. M. (2005). Sensor-directed response surface sampling designs for characterizing spatial variation in soil properties. Computers and Electronics in Agriculture, 46(1-3), 153-179.

Lesch, S., Strauss, D. J., \& Rhoades, J. D. (1995). Spatial Prediction of Soil Salinity Using Electromagnetic Induction Techniques: 2. An Efficient Spatial Sampling Algorithm Suitable for Multiple Linear Regression Model Identification and Estimation. Water Resources Research, 31(2), 387.

Murisier, F., \& Zufferey, V. (1997). Rapport feuille-fruit de la vigne et qualite du raisin. (Effect of the balance leave-fruit in vine plants and grape quality.) Revue Suisse de Viticulture, Arboriculture, Horticulture, 29, 355-362.

Ojeda, H., Carrillo, N., Deis, L., Tisseyre, B., Heywang, M., \& Carbonneau, A. (2005). Precision viticulture and water status II: Quantitative and qualitative performance of different within field zones, defined from water potential mapping. In H. R. Schultz (Ed.), Proceedings of 14th GESCO congress (pp. 741-748). Geisenheim, Germany: Groupe d'Etudes des Systemes de Conduite de la Vigne.

Onoyama, H., Ryu, C., Suguri, M., \& Iida, M. (2015). Nitrogen prediction model of rice plant at panicle initiation stage using ground-based hyperspectral imaging: growing degree-days integrated model. Precision Agriculture, 16(5), 558-570.

Samani Majd, A. M., Bleiweiss, M. P., DuBois, D., \& Shukla, M. K. (2013). Estimation of the fractional canopy cover of pecan orchards using Landsat 5 satellite data, aerial imagery, and orchard floor photographs. International Journal of Remote Sensing, 34(16), 5937-5952.

Santesteban, L. G., Miranda, C., \& Royo, J. B. (2011). Regulated deficit irrigation effects on growth, yield, grape quality and individual anthocyanin composition in Vitis vinifera L. cv. “Tempranillo.” Agricultural Water Management, 98(7), 1171-1179.

Santesteban, L. G., \& Royo, J. B. (2006). Water status, leaf area and fruit load influence on berry weight and sugar accumulation of cv. 'Tempranillo' under semiarid conditions. Scientia Horticulturae, $109(1), 60-65$.

Scholander, P. F., Bradstreet, E. D., Hemmingsen, E. a, \& Hammel, H. T. (1965). Sap pressure in vascular plants. Science, 148(3668), 339-46.

Sibille, I., Ojéda, H., Prieto, J., Maldonado, S., Ladapère, J. N., \& Carbonneau, A. (2007). Rapport entre les trois modalités de la chambre à pression (potentiel hydrique foliaire de base, foliaire à midi et «de tige» à midi) en fonction de la réponse de quatre cépages dans le sud de la France, applications pour le contrôle de l'irrigation. (Relation between the values of three pressure chamber modalities (midday leaf, midday stem and predawn water potential) of 4 grapevine cultivars in drought situation of the southern of France. Applications for the irrigation control). In Proceedings of 15th GESCO congress (pp. 685-695). Porec, Croatia: Institut za poljoprivredu i turizam.

Taylor, J. A., Acevedo-Opazo, C., Ojeda, H., \& Tisseyre, B. (2010). Identification and significance of sources of spatial variation in grapevine water status. Australian Journal of Grape and Wine Research, 16(1), 218-226.

Tisseyre, B., Mazzoni, C., \& Fonta, H. (2008). Whithin-field temporal stability of some parameters in viticulture: Potential toward a site specific management. Journal International Des Sciences De La Vigne Et Du Vin, 42(1), 27-39. 
Author-produced version of the article published in Precision Agriculture, 2017, $\mathrm{N}^{\circ}($ ), p.

The original publication is available at https://link.springer.com

http://dx.doi.org/10.1007/s11119-017-9523-8

Wulfsohn D. (2010). Sampling Techniques for Plants and Soil. Landbauforschung Völkenrode, (Special Issue 340), 3-30.

Zarrouk, O., Francisco, R., Pinto-Marijuan, M., Brossa, R., Santos, R. R., Pinheiro, C., et al. (2012). Impact of irrigation regime on berry development and flavonoids composition in Aragonez (Syn. Tempranillo) grapevine. Agricultural Water Management, 114, 18-29. 